\title{
\begin{tabular}{l|l} 
Mibraries & DSpace@MIT
\end{tabular}
}

\author{
MIT Open Access Articles
}

\section{Microstructural Rearrangements and their Rheological Implications in a Model Thixotropic Elastoviscoplastic Fluid}

The MIT Faculty has made this article openly available. Please share how this access benefits you. Your story matters.

Citation: Jamali, Safa, Gareth H. McKinley, and Robert C. Armstrong. “Microstructural Rearrangements and Their Rheological Implications in a Model Thixotropic Elastoviscoplastic Fluid." Physical Review Letters 118.4 (2017): n. pag. (c) 2017 American Physical Society.

As Published: http://dx.doi.org/10.1103/PhysRevLett.118.048003

Publisher: American Physical Society

Persistent URL: http://hdl.handle.net/1721.1/107486

Version: Final published version: final published article, as it appeared in a journal, conference proceedings, or other formally published context

Terms of Use: Article is made available in accordance with the publisher's policy and may be subject to US copyright law. Please refer to the publisher's site for terms of use. 


\title{
Microstructural Rearrangements and their Rheological Implications in a Model Thixotropic Elastoviscoplastic Fluid
}

\author{
Safa Jamali, ${ }^{1}$ Gareth H. McKinley, ${ }^{2}$ and Robert C. Armstrong ${ }^{1}$ \\ ${ }^{1}$ Department of Chemical Engineering, Massachusetts Institute of Technology, \\ 77 Massachusetts Avenue, Cambridge, Massachusetts 02139, USA \\ ${ }^{2}$ Department of Mechanical Engineering, Massachusetts Institute of Technology, \\ 77 Massachusetts Avenue, Cambridge, Massachusetts 02139, USA
}

(Received 7 July 2016; revised manuscript received 1 November 2016; published 27 January 2017)

\begin{abstract}
We identify the sequence of microstructural changes that characterize the evolution of an attractive particulate gel under flow and discuss their implications on macroscopic rheology. Dissipative particle dynamics is used to monitor shear-driven evolution of a fabric tensor constructed from the ensemble spatial configuration of individual attractive constituents within the gel. By decomposing this tensor into isotropic and nonisotropic components we show that the average coordination number correlates directly with the flow curve of the shear stress versus shear rate, consistent with theoretical predictions for attractive systems. We show that the evolution in nonisotropic local particle rearrangements are primarily responsible for stress overshoots (strain-hardening) at the inception of steady shear flow and also lead, at larger times and longer scales, to microstructural localization phenomena such as shear banding flow-induced structure formation in the vorticity direction.
\end{abstract}

DOI: 10.1103/PhysRevLett.118.048003

Thixotropic elastoviscoplastic (TEVP) materials are a broad class of structured fluids that include (but are not limited to) most colloidal gels [1], nano emulsions [2], crude oils $[3,4]$, and many biological systems such as blood clots and actin networks $[5,6]$. As a result of their complex underlying microstructure, TEVPs exhibit a wide range of rich and complex thermo-mechanical properties: Below a critical stress, the microstructural network formed by individual particles remains intact and resists large deformations by external forces. At this stage the macroscopic response of the material is similar to that of a viscoelastic solid. By progressively increasing the applied load, the material reaches its "yield stress" and starts to flow [7]. At this point the particle network that is responsible for solidlike response of the macroscopic sample undergoes plastic rearrangements over an increasingly wide range of length scales [8]. Upon complete yielding of this network, plastic flow results ultimately in a viscouslike response; however, as a result of constant formation and breakage events, the particle-level microstructure continues to evolve giving rise to thixotropic behavior. The many-body nature of the problem means that local forces exerted on a single particle change its energy landscape, which consequently defines its subsequent association or dissociation rate to neighboring particles $[9,10]$. When combined with multibody hydrodynamic effects in these fluids [11], the resulting microstructure-flow relationship becomes complex and may show long time scale transient behavior and multiple steady states $[1,12]$. This leads to a wide range of timedependent responses that can also be observed, including microphase separation [13], vorticity aligned structure formation [14-16], local rigid plug formation and shear banding [17], plus shear-induced rejuvenation of the particle network [18].

Although the general form of the flow curve (relating the shear stress to shear rate) and some transient phenomena of TEVP fluids can be described by detailed continuum models [3,19-21], a clear and fundamental understanding of the underlying mechanisms requires connecting macroscopic material properties to events that happen at the microstructural level. Experimentally, making this connection is very challenging because monitoring of the entire microstructure under flow is essential, together with elaborate protocols to produce well-defined initial conditions [22]. Thus, numerical simulations can play a crucial role in providing a detailed picture of the local structural evolution in these complex fluids.

Bridging the gap between the particle-level interactions and the macroscopic rheological response of a complex fluid requires access to mesoscopic length and time scales spanning the microscale, where the building blocks of a system are described explicitly, while concomitantly accessing much longer time or length scales relevant to macroscopic phenomena. To span this wide range of length or time scales, we perform dissipative particle dynamics (DPD) [23] simulations on model TEVP fluids consisting of $10 \mathrm{vol} \%$ attractive hexagonal solid particles designed to represent the waxy crystalline particles observed in a waxy crude oil $[3,24]$. The DPD formalism inherently preserves multibody hydrodynamics by conservation of mass and momentum both locally and globally $[25,26]$. Furthermore, by incorporating relevant interaction potentials for 
attractive colloids, this technique can provide a realistic representation of a TEVP fluid.

In DPD, the equation of motion of a particle is written based on pairwise interaction potentials $m_{i}\left(d \mathbf{v}_{i} / d t\right)=$ $\sum_{j=1}^{N} \mathbf{F}_{i j}^{D}+\mathbf{F}_{i j}{ }^{R}+\mathbf{F}_{i j}{ }^{C}$, which act on each individual particle [27]. Here, $N$ is the number of interacting neighbors around particle $i$. In traditional DPD [23,27-29], the conservative force is written as a soft linearly decaying repulsive potential, $\mathbf{F}_{i j}^{C}=a_{i j} \omega_{i j}^{C}\left(r_{i j}\right) \mathbf{e}_{i j}$. The attractive nature of local particle-particle interactions in TEVP materials demands that a modified conservative potential be employed [30]. Thus, we modify the interaction potential between the solid particles to a Morse potential $\mathbf{F}_{i j}^{C}=\Gamma \kappa\left(e^{-\kappa r_{i j}}-e^{-2 \kappa r_{i j}}\right) \mathbf{e}_{i j}$, where $\Gamma$ is the depth of the attraction well and $\kappa^{-1}$ is the characteristic range of attraction [30]. Because of the short-range ( 0.2 of the particle size) nature of the attractive potential, $\Gamma$ provides a direct measure of the attraction strength between the particles. We have performed simulations with the attraction strength of $3 k_{B} T \leq \Gamma \leq 50 k_{B} T$, representing a range of weak to strong interactions between the particles with $\kappa^{-1}=0.15$.

In order to ensure that the prior thermomechanical history does not affect the rheological measures, simulations were initiated with randomly distributed particles with no flow or temperature history. Under quiescent conditions these
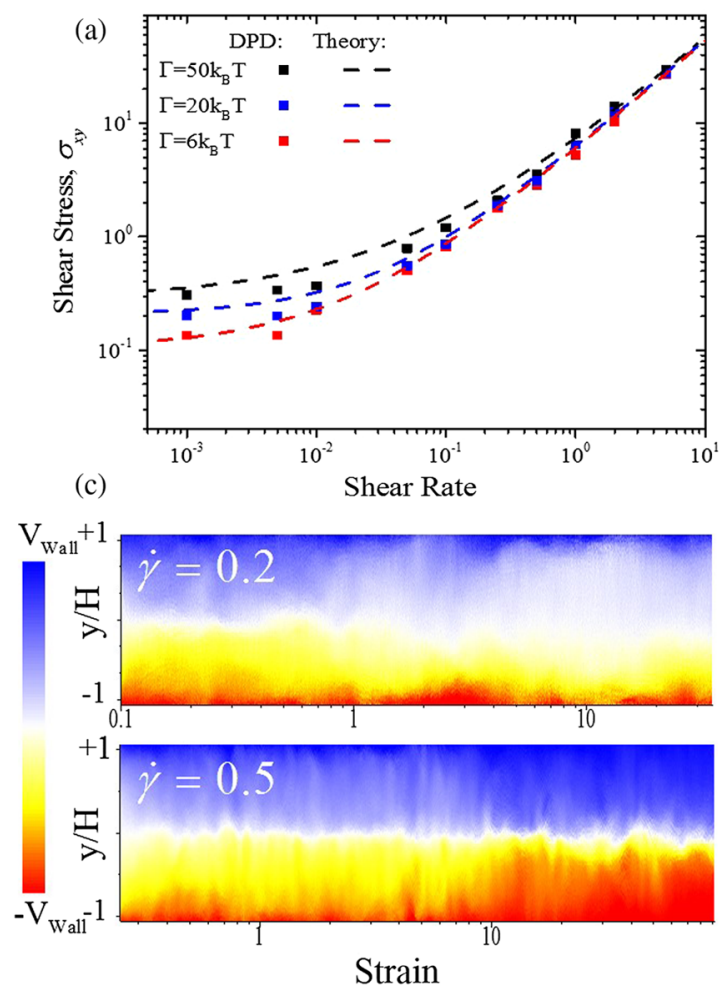

Brownian attractive particles self-assemble into spacespanning networks whose fractal structure was examined via the box-counting method (see Supplemental Material [31], which includes Refs. [3,4,24,25,27,32-36]). These gel networks were then subject to an imposed shear flow, using Lees-Edwards boundary conditions (constant velocity of the upper and lower boundary cells, induces a linear velocity profile within the calculation box for a simple fluid with $\dot{\gamma}=2 V_{\text {Wall }} / L_{y}$ ) $[34,35]$. We have performed simulations with several different initial microstructures of the gelled network and find that when driven out of equilibrium, the resulting microstructure or rheology is dominated by the imposed shear rate and total strain, and is independent of the initial microstructures.

We evaluate the steady state value of the corresponding shear stress $\sigma_{x y}$ at long times, and Fig. 1 shows the general flow curve for an attractive gel $\left(\phi=0.1\right.$ and $\left.\Gamma=20 k_{B} T\right)$, as well as the initial evolution of shear stress as a function of accumulated strain deformation. The first noticeable feature of Fig. 1(a) is that the key experimentally observed feature $[3,4]$ corresponding to yielding behavior in a TEVP gel is reproduced: the shear stress approaches a finite plateau at low dimensionless values of the imposed shear rate $\dot{\gamma} \leq 0.1$. This is in agreement with theoretical predictions of Snabre and Mills [20] for attractive colloidal

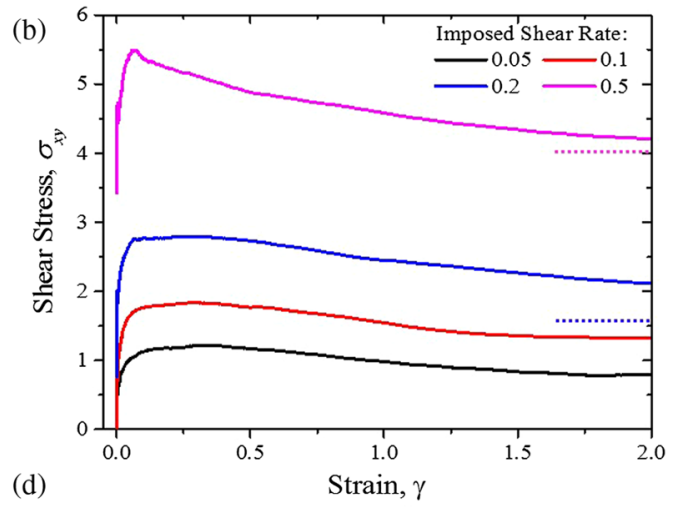

(d)

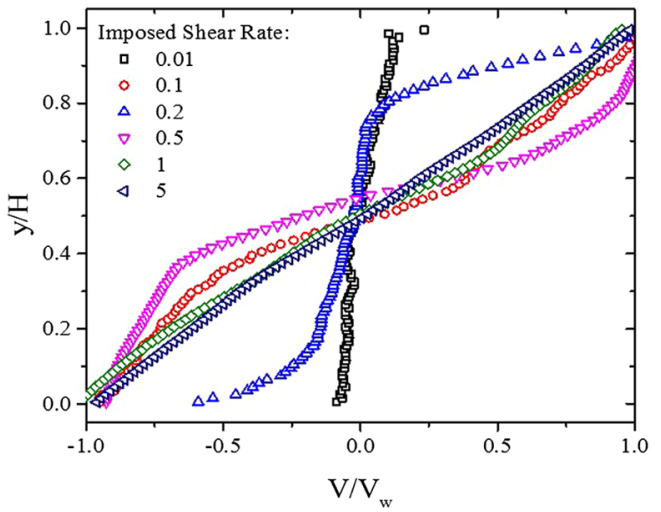

FIG. 1. (a) Shear stress versus shear rate, compared to Ref. [20]. (b) Stress overshoot response in flow start-up simulations for different shear rates; dashed lines show the steady state asymptotes at sufficiently large deformations. (c) Space-time diagrams of the velocity field $v_{x}(y, t)$ in the gel under two different strain rates showing flow instability and spatial inhomogeneities at large strains or times, and (d) final averaged velocity profiles across the calculation box $\bar{v}_{x}(y)$ for a range of shear rates. 
systems under steady shear flow. Figure 1(b) shows the time-dependent flow behavior of the gels at the start-up of steady shear, where the shear stress is plotted as a function of imposed strain for different shear rates. A marked stress overshoot is evident regardless of the imposed shear rate at a dimensionless strain of $\gamma \simeq 0.2$. This is consistent with several experimental measurements $[13,37,38]$ and computational predictions of these materials at flow start-up $[8,39]$. The origin of this stress overshoot is commonly associated with rearrangement of particles within the fluid in conjunction with stretching of the microstructure along the direction of maximum extension [8]. Numerical simulations of viscoelastic constitutive equations have also shown that stress overshoots are commonly associated with the onset of hydrodynamic instabilities and the development of inhomogeneous shearing profiles in shear complex fluids [17,40,41]. In Fig. 1(c) we show space-time diagrams of the velocity profiles $v_{x}(y, t)$ across the simulation cell, together with final profiles at $t=250$ presented in Fig. 1(d) for a range of shear rates. The profiles in Fig. 2(d) are clearly not linear across the box and show strong evidence of flow inhomogeneity at intermediate deformation rates, $0.1<\dot{\gamma}<1.0$. At smaller deformation rates, $\dot{\gamma}<0.1$, significant slip is observed with regards to velocity of the moving boundaries, whereas at the highest rates of $\dot{\gamma}>1$ no evidence of inhomogeneity is observed across the calculation cell. We seek to understand how evolution of the material on the microscale gives rise to these observed kinematic inhomogeneities.

To do so, we characterize the microstructure of the gel network through the fabric tensor $\mathbf{Z}$ [42-44]. As two interacting particles approach within the attraction range, they form particle bonds. To monitor the collective dynamics of the system we define $\mathbf{Z}$ from the local fabric [42-44] of single particles $\left(\mathbf{Z}^{p}=\sum_{i} \mathbf{n}_{i} \otimes \mathbf{n}_{i}\right)$, where $\mathbf{n}_{i}$ is the unit vector of the center-center line between a particle and its $i$ th bond forming neighbor. Averaging $\mathbf{Z}^{p}$ over the particle ensemble will yield a system-sized fabric tensor: $\mathbf{Z}=(1 / N) \sum_{p=1}^{N} \mathbf{Z}^{p}$. The trace of this ensemble fabric tensor provides the average number of bonds in a system

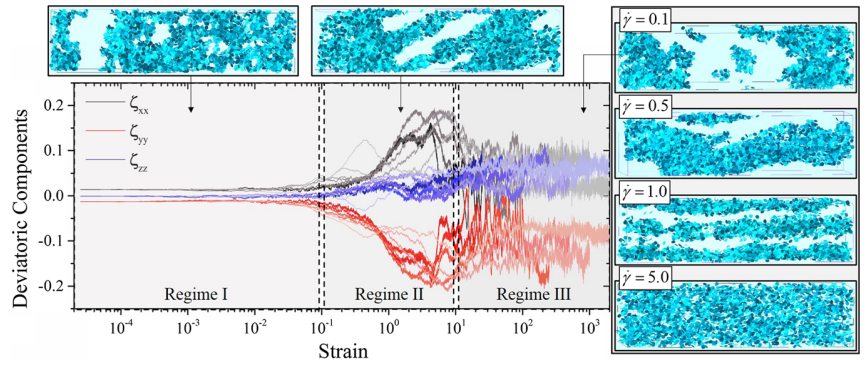

FIG. 2. Deviatoric components of the fabric tensor as a function of accumulated strain for different shear rates (color increments from dark to light indicate increase in shear rate), accompanied with snapshots of representative microstructures in different strain regimes. $\operatorname{tr} \mathbf{Z} \equiv\langle Z\rangle$, i.e., the coordination number, or the isotropic scalar defining the configuration of each particle and its nearest neighbors $[22,39,45]$. The deviatoric components of this fabric tensor can provide significant information about evolution in the anisotropy of the structure under different conditions. We define the deviatoric measure as $\zeta=$ $\mathbf{Z}-(\operatorname{tr} \mathbf{Z} / 3) \mathbf{I}$, where $\mathbf{I}$ is the identity tensor. The temporal evolution of the deviatoric components of the fabric tensor are presented in Fig. 2, as a function of the accumulated strain for different dimensionless shear rates. In our notation the $x$, $y$, and $z$ components correspond to the flow, the velocity gradient, and the vorticity directions, respectively. The microstructural insights from the evolution of the fabric tensor in Fig. 2 can be separated into three distinct regimes. (i) Upon flow start-up, and within the linear viscoelastic regime (at small strains of $\gamma \leq 0.1$ ), the particulate network remains rather isotropic with little change in the microstructure and the corresponding stress grows linearly with strain [Fig. 1(b)]. (ii) At $\gamma \simeq 0.1$ the material response deviates from linear viscoelasticity, and the increase in the $\zeta_{x x}$ component suggests that the interparticle bonds start to rearrange preferentially into the $x$ direction. At these deformation levels of $0.1 \leq \gamma \leq 10$, bonds are also increasingly broken in the $y$ direction, as evident from the decrease in $\boldsymbol{\zeta}_{y y}$. (iii) At larger strains of $\gamma \geq 10$, persistent transient structures are observed, with both $\zeta_{x x}$ and $\zeta_{y y}$ showing steady plateaus (after passing through a maximum and a minimum respectively), and, in addition, $\boldsymbol{\zeta}_{z z}$ starts to increase, indicating the slow evolution of structures aligned in the vorticity direction.

It should be noted that although the general evolution in the microstructural anisotropies are similar regardless of imposed strain rates, the morphology of the particulate network on larger length scales varies significantly depending on the rate of shearing. This is evident in the final images shown for $\gamma \simeq 100$ in Fig. 2. At high shear rates $(\dot{\gamma}=5)$ the microstructure becomes homogeneous, whereas at lower deformation rates of $0.1,0.5$, and 1 , small clusters, pluglike phase separated morphologies, and stringlike layers of particles are observed, respectively.

Figure 3 shows the evolution in the off-diagonal shear component of the fabric tensor $Z_{x y}$ as well as the coordination number $\operatorname{tr} \mathbf{Z}=\langle Z\rangle$ as functions of shear rate and accumulated strain. As evident in Fig. 3(a) the shear component of the fabric tensor (Fig. 3) follows the shear stress regardless of the applied deformation rate, and undergoes an overshoot at $\gamma \simeq 1$. As expected, this confirms that the deviation in linear viscoelastic response of a TEVP fluid originates from the rearrangements occurring within its microstructure. In addition, the other nondiagonal components show similar universal changes for the same range of strains (see Fig. S8 in the Supplemental Material [31]).

However, following the trace of the fabric tensor trZ for the same range of shear rates allows us to clearly differentiate between different material responses [Fig. 3(b)]. At low strain rates, prolonged shearing results 


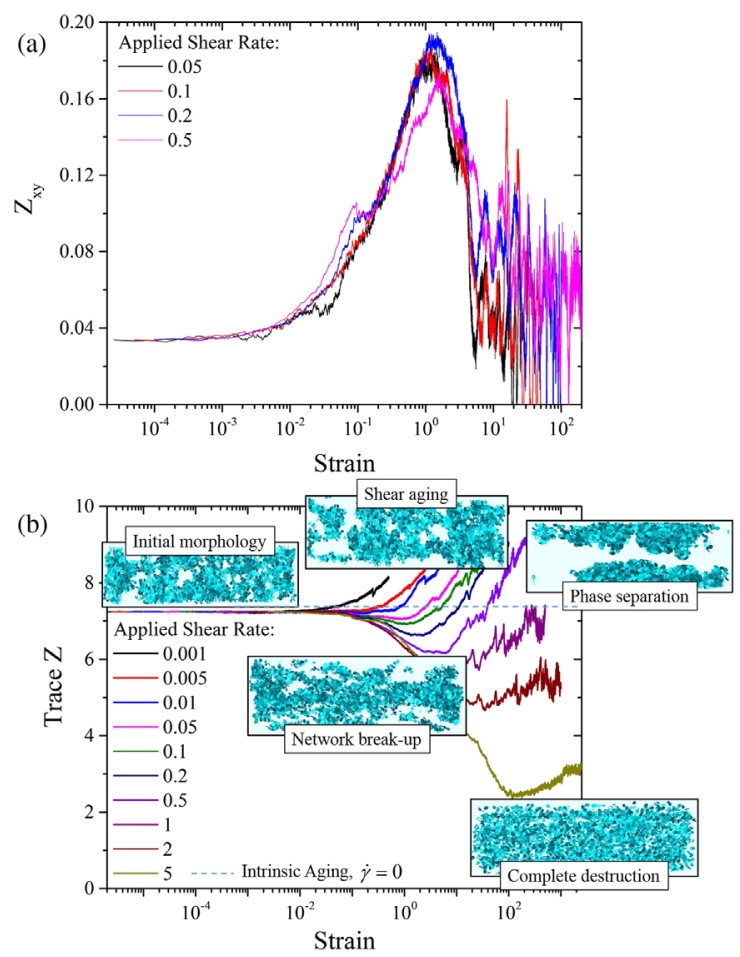

FIG. 3. (a) Overshoot in the shear component of the fabric tensor in flow start-up simulations, and (b) trace of the fabric tensor (coordination number) for different shear rates. Insets show representative microstructures in different flow regimes (compaction, shear rejuvenation, network breakup, etc.).

in a net formation of new bonds and compaction of the structure, and thus this regime is most commonly referred to as "shear aging" [30], and trZ increases monotonically. By increasing the shear rate above the critical strain rate for macroscopic yielding, the shear stresses initially break the network into smaller clusters and individual particles, resulting in a steady decrease in the coordination number. However, new intracluster bonds form on longer time scales, and this results in larger coordination numbers than the initial configuration as these secondary structures begin to form. This regime is most commonly referred to as shear rejuvenation [46], as the initial structure is progressively destroyed and a new one is formed. For high accumulated deformations, large secondary structures are formed, which are highly anisotropic (as confirmed by the individual diagonal components of the fabric tensor presented in Fig. 2 and Fig. S8 in the Supplemental Material [31]). At the highest shear rates $\dot{\gamma} \geq 5$, the macroscopic response corresponds to steady viscoplastic flow [Fig. 1(a)] and the particulate network is effectively broken into small homogenous structures with very low coordination numbers.

Heterogeneities in the microstructure of the fluid under flow result in inhomogeneous fabric tensor measurements at the particle level. Thus, in addition to presenting ensemble-averaged measures of the fabric components as shown in Figs. 2 and 3, we also show in Fig. 4 the
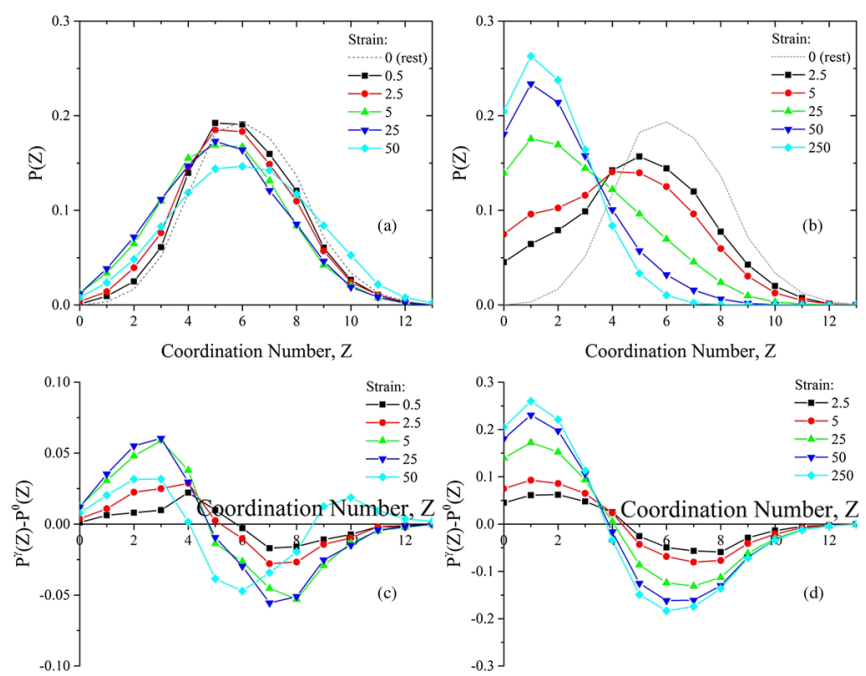

FIG. 4. Evolution in the distribution of coordination number for a network of 4500 attractive particles $\left(\phi=0.1\right.$ and $\left.\Gamma=20 k_{B} T\right)$ at different strains under shear rates of 0.5 (a) and 5.0 (b). Change in the coordination number relative to the quiescent distribution for a shear rate of 0.5 (c) and 5.0 (d).

distribution of coordination numbers in the fluid compared to this distribution prior to inception of flow.

Examining the distribution of the coordination numbers for the gel that undergo breakup and reformation of transient structures at intermediate shear rate of 0.5 in Figs. 4(a), 4(c) shows that at short times $(\gamma<10)$ the population of particles with $Z \geq 6$ progressively decreases in the system and the number of particles with $Z \leq 5$ increases. However, at longer times when secondary structures are formed, the coordination number distribution relative to the quiescent condition $P^{\gamma}(Z)-P^{0}(Z)$ shows two maxima and a minimum, indicating that there are local flocculated subdomains, coexistent with regions in which the particles have smaller coordination numbers. However, by increasing the shear rate to 5.0, a gradual destruction of the particle network is observed, in agreement with results presented in Fig. 3, and with the experimental measurements by Hsiao et al. [22].

In summary, we have shown that the particle networks reproduced by DPD for soft attractive particles capture the key rheological features of a model TEVP fluid and are consistent with the predicted form of phenomenological models for attractive systems. Calculating a fabric tensor from the ensemble of individual particle-particle bonds within a gel-forming colloidal system provides additional insight about the microstructural changes that occur under flow. Particle rearrangements at moderate strains, $0.1 \leq \gamma \leq 2$, lead to an overshoot in the shear stress and the $Z_{x y}$ component of the fabric tensor also undergoes a similar overshoot. To decouple changes in the average isotropic and anisotropic contributions to the microstructure of the attractive gel under shear we calculate the deviatoric fabric tensor, $\zeta$. This shows evidence of bond rearrangements 
in the flow direction and the velocity gradient direction, resulting in a net positive value of $\zeta_{x x}-\zeta_{y y}$. At higher strain units, $\gamma \geq 10$, our three-dimensional calculations of $\boldsymbol{\zeta}_{z z}$ also show development of secondary structure formation in the vorticity direction. This coincides with shear rejuvenation and a decrease in the average coordination number of the microstructure [Fig. 3(b)] and in the development of the strongly inhomogeneous shearing velocity profiles we have documented in the space-time diagrams [Figs. 1(c), 1(d)].

Our results demonstrate that the DPD method can provide a systematic route to bridging the gap between direct observations [22] of microstructure and measurements of the macroscopic rheological response of a TEVP fluid to an imposed deformation rate. Our steady-state simulations show quantitative agreement with closed-form constitutive equations for yield stress fluids, but such models provide no insight into transient responses of such materials. Recent microscopic measurements, as well as large scale numerical simulations, have begun to focus on the start-up of steady shear flow and have clearly documented the evolution of particulate level microstructure under deformation $[8,18,22,39,45,47,48]$. The wide range of time and length scales accessible by DPD simulations, coupled with inclusion of hydrodynamic interactions between particles [25,49], now enables systematic exploration of the evolution of microstructural and rheological properties for TEVP materials as well as validation of empirical constitutive equations proposed for such systems [21,50-53].

[1] E. Zaccarelli, Colloidal gels: equilibrium and non-equilibrium routes, J. Phys. Condens. Matter 19, 323101 (2007).

[2] M. E. Helgeson, Y. Gao, S. E. Moran, J. Lee, M. Godfrin, A. Tripathi, A. Bose, and P. S. Doyle, Homogeneous percolation versus arrested phase separation in attractively-driven nanoemulsion colloidal gels, Soft Matter 10, 3122 (2014).

[3] C. J. Dimitriou and G. H. McKinley, A comprehensive constitutive law for waxy crude oil: A thixotropic yield stress fluid, Soft Matter 10, 6619 (2014).

[4] C. J. Dimitriou, G. H. McKinley, and R. Venkatesan, RheoPIV analysis of the yielding and flow of model waxy crude oils, Energy Fuels 25, 3040 (2011).

[5] C. Storm, J. J. Pastore, F. C. MacKintosh, T. C. Lubensky, and P. A. Janmey, Nonlinear elasticity in biological gels, Nature (London) 435, 191 (2005).

[6] M. L. Gardel et al., Elastic behavior of cross-linked and bundled actin networks, Science 304, 1301 (2004).

[7] D. Bonn et al., Yield stress materials in soft condensed matter, arXiv:1502.05281.

[8] J. Colombo and E. Del Gado, Stress localization, stiffening, and yielding in a model colloidal gel, J. Rheol. 58, 1089 (2014).

[9] E. Evans and K. Ritchie, Dynamic strength of molecular adhesion bonds, Biophys. J. 72, 1541 (1997).

[10] L. C. Hsiao, M. J. Solomon, K. A. Whitaker, and E. M. Furst, A model colloidal gel for coordinated measurements of force, structure, and rheology, J. Rheol. 58, 1485 (2014).
[11] D. R. Foss and J. F. Brady, Structure, diffusion and rheology of Brownian suspensions by Stokesian Dynamics simulation, J. Fluid Mech. 407, 167 (2000).

[12] J. Vermant and M. J. Solomon, Flow-induced structure in colloidal suspensions, J. Phys. Condens. Matter 17, R187 (2005).

[13] B. Rajaram and A. Mohraz, Microstructural response of dilute colloidal gels to nonlinear shear deformation, Soft Matter 6, 2246 (2010).

[14] T. Gibaud, C. Barentin, N. Taberlet, and S. Manneville, Shear-induced fragmentation of laponite suspensions, Soft Matter 5, 3026 (2009).

[15] V. Grenard, N. Taberlet, and S. Manneville, Shear-induced structuration of confined carbon black gels: steady-state features of vorticity-aligned flocs, Soft Matter 7, 3920 (2011).

[16] C. O. Osuji and D. A. Weitz, Highly anisotropic vorticity aligned structures in a shear thickening attractive colloidal system, Soft Matter 4, 1388 (2008).

[17] S. M. Fielding, Shear banding in soft glassy materials, Rep. Prog. Phys. 77, 102601 (2014).

[18] J. Colombo, A. Widmer-Cooper, and E. Del Gado, Microscopic Picture of Cooperative Processes in Restructuring Gel Networks, Phys. Rev. Lett. 110, 198301 (2013).

[19] P. Snabre and P. Mills, II. Rheology of weakly flocculated suspensions of viscoelastic particles, J. Phys. III (France) 6, 1835 (1996).

[20] P. Snabre and P. Mills, I. Rheology of weakly flocculated suspensions of rigid particles, J. Phys. III (France) 6, 1811 (1996).

[21] P. R. de Souza Mendes, Thixotropic elasto-viscoplastic model for structured fluids, Soft Matter 7, 2471 (2011).

[22] L. C. Hsiao, R. S. Newman, S. C. Glotzer, and M. J. Solomon, Role of isostaticity and load-bearing microstructure in the elasticity of yielded colloidal gels, Proc. Natl. Acad. Sci. U.S.A. 109, 16029 (2012).

[23] P. J. Hoogerbrugge and J. M. V. A. Koelman, Simulating microscopic hydrodynamic phenomena with dissipative particle dynamics, Europhys. Lett. 19, 155 (1992).

[24] M. Kané, M. Djabourov, J.-L. Volle, J.-P. Lechaire, and G. Frebourg, Morphology of paraffin crystals in waxy crude oils cooled in quiescent conditions and under flow, Fuel 82, 127 (2003).

[25] P. Espanol, Hydrodynamics from dissipative particle dynamics, Phys. Rev. E 52, 1734 (1995).

[26] T. Zhao, X. Wang, L. Jiang, and R. G. Larson, Dissipative particle dynamics simulation of dilute polymer solutionsInertial effects and hydrodynamic interactions, J. Rheol. 58, 1039 (2014).

[27] R. D. Groot and P. B. Warren, Dissipative particle dynamics: Bridging the gap between atomistic and mesoscopic simulation, J. Chem. Phys. 107, 4423 (1997).

[28] P. J. Hoogerbrugge and J. M. V. A. Koelman, Dynamic simulations of hard-sphere suspensions under steady shear, Europhys. Lett. 21, 363 (1993).

[29] C. A. Marsh, G. Backx, and M. H. Ernst, Fokker-PlanckBoltzmann equation for dissipative particle dynamics, Europhys. Lett. 38, 411 (1997).

[30] R. N. Zia, B. J. Landrum, and W. B. Russel, A micromechanical study of coarsening and rheology of colloidal 
gels: Cage building, cage hopping, and Smoluchowski's ratchet, J. Rheol. 58, 1121 (2014).

[31] See Supplemental Material at http://link.aps.org/ supplemental/10.1103/PhysRevLett.118.048003 for details of simulation parameters, gelation under quiescent conditions and particle-level fabric analysis, which includes Refs. [3,4,24,25,27,32-36].

[32] P. Espanol and P. Warren, Statistical mechanics of dissipative particle dynamics, Europhys. Lett. 30, 191 (1995).

[33] S. Jamali, A. Boromand, S. Khani, J. Wagner, M. Yamanoi, and J. Maia, Generalized mapping of multi-body dissipative particle dynamics onto fluid compressibility and the FloryHuggins theory, J. Chem. Phys. 142, 164902 (2015).

[34] A. Boromand, S. Jamali, and J. M. Maia, Viscosity measurement techniques in dissipative particle dynamics, Comput. Phys. Commun. 196, 149 (2015).

[35] A. W. Lees and S.F. Edwards, The computer study of transport processes under extreme conditions, J. Phys. C 5, 1921 (1972).

[36] S. Jamali, A. Boromand, S. Khani, and J. Maia, Gaussianinspired auxiliary non-equilibrium thermostat (GIANT) for dissipative particle dynamics simulations, Comput. Phys. Commun. 197, 27 (2015).

[37] A. Mohraz and M. J. Solomon, Orientation and rupture of fractal colloidal gels during start-up of steady shear flow, J. Rheol. 49, 657 (2005).

[38] N. Koumakis and G. Petekidis, Two step yielding in attractive colloids: transition from gels to attractive glasses, Soft Matter 7, 2456 (2011).

[39] J. D. Park and K. H. Ahn, Structural evolution of colloidal gels at intermediate volume fraction under start-up of shear flow, Soft Matter 9, 11650 (2013).

[40] S. M. Fielding, Complex dynamics of shear banded flows, Soft Matter 3, 1262 (2007).

[41] S. M. Fielding and P. D. Olmsted, Spatiotemporal Oscillations and Rheochaos in a Simple Model of Shear Banding, Phys. Rev. Lett. 92, 084502 (2004).
[42] T. Olsen and K. Kamrin, Modeling tensorial conductivity of particle suspension networks, Soft Matter 11, 3875 (2015).

[43] M. M. Mehrabadi, S. Nemat-Nasser, and M. Oda, On statistical description of stress and fabric in granular materials, Int. J. Numer. Anal. Methods Geomech. 6, 95 (1982).

[44] M. Oda, S. Nemat-Nasser, and M. M. Mehrabadi, A statistical study of fabric in a random assembly of spherical granules, Int. J. Numer. Anal. Methods Geomech. 6, 77 (1982).

[45] A. Mohraz and M. J. Solomon, Orientation and rupture of fractal colloidal gels during start-up of steady shear flow, J. Rheol. 49, 657 (2005).

[46] P. Coussot, Q. D. Nguyen, H. T. Huynh, and D. Bonn, Avalanche Behavior in Yield Stress Fluids, Phys. Rev. Lett. 88, 175501 (2002).

[47] J. Colombo and E. Del Gado, Self-assembly and cooperative dynamics of a model colloidal gel network, Soft Matter 10, 4003 (2014).

[48] X. Cheng, J. H. McCoy, J. N. Israelachvili, and I. Cohen, Imaging the microscopic structure of shear thinning and thickening colloidal suspensions, Science 333, 1276 (2011).

[49] X. Bian, C. Kim, and G. Karniadakis, 111 years of Brownian motion, Soft Matter 12, 6331 (2016).

[50] M. J. Armstrong, A. N. Beris, S. A. Rogers, and N. J. Wagner, Dynamic shear rheology of a thixotropic suspension: Comparison of an improved structure-based model with large amplitude oscillatory shear experiments, J. Rheol. 60, 433 (2016).

[51] R. G. Larson, Constitutive equations for thixotropic fluids, J. Rheol. 59, 595 (2015).

[52] K. Dullaert and J. Mewis, A model system for thixotropy studies, Rheol. Acta 45, 23 (2005).

[53] J. Mewis and N. J. Wagner, Thixotropy, Adv. Colloid Interface Sci. 147-148, 214 (2009). 